\title{
APPENDIX II. X-RAY MINERALOGICAL ANALYSIS OF CRETACEOUS SEQUENCES, LEG 80 (GOBAN SPUR, SITES 548, 549, 550, 551) ${ }^{1}$
}

\author{
M. Thiry, École Nationale Supérieure des Mines, Paris \\ and \\ A. Pascal, Institut des Sciences de la Terre de l'Université de Dijon
}

\section{INTRODUCTION}

At the request of the Leg 80 scientific party, selected samples of Cretaceous age were processed by X-ray diffraction at the mineralogy laboratories at the École des Mines (Albian to Late Cretaceous samples) and at the Institut de Géologie at Dijon (Barremian samples). The results of these mineralogical analyses are shown in Tables 1 and 2 and Figures 1 to 3 . The results were used in developing the lithostratigraphy and sedimentology discussed in this volume by Rat et al. in their study of Barremian-Albian paleoenvironment, by Graciansky and Gillot in their study of Albian and Cenomanian limestones, and by Graciansky and Bourbon in their paleoenvironmental reconstructions for the Late Cretaceous chalks.

\section{METHODS}

The bulk sediment samples were dried and pulverized with a mortar of agate and then analyzed according to the powder diagram method. The contents of the various components were estimated from the diagrams by

\footnotetext{
${ }^{1}$ Graciansky, P. C. de, Poag, C. W., et al., Init. Repts. DSDP, 80: Washington (U.S. Govt, Printing Office).

2 Addresses: (Thiry) École Nationale Supérieure des Mines, F77305 Fontainebleau, France: (Pascal) Institut des Sciences de la Terre de I'Université, 6 Boulevard Gabriel F2110 Dijon, France.
}

comparing the intensity of the characteristic peaks, taking into account the differences between the intensities of diffraction of the various minerals. Estimates were related to a total of $100 \%$ to minimize the effects of the matrix and of the influence of the preparation of the powders. The estimates of the total amount of clay minerals and of cristobalite and tridymite is relatively uncertain because of the low crystallinity of these minerals.

To prepare the clay fraction, the bulk sediments were dispersed in pure water by mechanical agitation. Samples were first treated with $\mathrm{HCl}$ mixed 1:10 with water. After several washings in the water, the $<2 \mu \mathrm{m}$ fractions were separated by settling. The $<2 \mu \mathrm{m}$ fractions were then centrifuged, and the thick paste obtained was spread across slides bearing a narrow groove. The oriented paste was then dried, saturated with ethylene glycol and hydrazine hydrate, and later heated at $490^{\circ}$. Quantitative estimates of the relative proportions of the clay minerals and the mixed-layer clays were obtained from the areas beneath the peaks in the powder diagrams for the samples treated by ethylene glycol.

Operating conditions involved using copper $\mathrm{K}$ radiation and a beam of $1^{\circ}$ of aperture for both the bulk sample and clay fraction analyses.

Date of Initial Receipt: November 18, 1983

Date of Acceptance: February 27, 1984

Table 1. X-ray analysis (\%) of bulk samples.

\begin{tabular}{|c|c|c|c|c|c|c|c|c|c|c|c|c|c|c|c|c|}
\hline $\begin{array}{l}\text { Core-Section } \\
\text { (interval or } \\
\text { level in } \mathrm{cm} \text { ) }\end{array}$ & Clays & Quartz & Calcite & Dolomite & $\begin{array}{l}\text { Plagio- } \\
\text { clase }\end{array}$ & $\begin{array}{l}\text { K-feld- } \\
\text { spars }\end{array}$ & Opal-CT & $\begin{array}{l}\text { Clinop- } \\
\text { tilolite }\end{array}$ & Pyrite & $\begin{array}{l}\text { Fluor- } \\
\text { apatite }\end{array}$ & Barite & Gypsum & Halite & $\begin{array}{c}\text { Not } \\
\text { identified }\end{array}$ & Lithology & Age \\
\hline \multicolumn{17}{|c|}{ Hole 548A } \\
\hline $29-1,36-42$ & & $t$ & 100 & & & & & & & & & & & & \multirow{11}{*}{$\begin{array}{l}\text { Foraminifer-nannofos- } \\
\text { sil chalks }\end{array}$} & \multirow{11}{*}{ Maestrichtian } \\
\hline $29-3,0-7$ & $\epsilon$ & $e ?$ & 100 & & & & & & & & ? & & $\epsilon$ & & & \\
\hline $30-1,103-104$ & $\epsilon$ & $\epsilon$ & 100 & & & & & & & & & & $\epsilon$ & & & \\
\hline $31-2,63-65$ & $\epsilon ?$ & e & 100 & & & & & & & & & & $\epsilon$ & & & \\
\hline $32-1,69-71$ & & $\epsilon$ & 100 & & & & & & & & & & $\epsilon$ & & & \\
\hline $32-4,69-71$ & & $\epsilon$ & 100 & & & & & & & & & & $\epsilon$ & & & \\
\hline $33-2,9-10$ & & $e$ & 100 & & & & & & & & & & . & & & \\
\hline $34-1,29-31$ & & & 100 & & & & & $e ?$ & & & & & $\epsilon$ & & & \\
\hline $34-3,29-31$ & 10 & e & 90 & & & & & & & & & & $\epsilon$ & & & \\
\hline $34-5,29-31$ & $\epsilon$ & $\epsilon$ & 100 & & & & & & & & & & $\epsilon$ & & & \\
\hline $34-6,29-31$ & $\epsilon$ & $\epsilon$ & 100 & & & & & ? & & & & & $\epsilon$ & & & \\
\hline \multicolumn{17}{|c|}{ Hole 549} \\
\hline $21-2,7-9$ & 25 & e & 75 & & & & & $\epsilon$ & & & $?$ & & & & \multirow{12}{*}{$\begin{array}{l}\text { Foraminifer-nannofos- } \\
\text { sil chalk }\end{array}$} & \multirow{12}{*}{$\begin{array}{l}\text { Coniac.- } \\
\text { Santon. }\end{array}$} \\
\hline $22-1,46-48$ & $?$ & e & 100 & & & & & $\epsilon$ & & & & & $t$ & & & \\
\hline $22-3,105-109$ & 10 & e & 90 & & & & & e & & & & & $t$ & & & \\
\hline $22-5,47-49$ & & $\epsilon$ & 100 & & & & & e? & & & & & $\epsilon$ & & & \\
\hline $23-2,52-54$ & & $\epsilon$ & 100 & & & & & $e ?$ & & & & & e & & & \\
\hline $23-4,50-52$ & & e & 100 & & & & & $e$ & & & & & e & & & \\
\hline $23-5,24-26$ & & e & 100 & & & & & $\epsilon ?$ & & & & & $\epsilon$ & & & \\
\hline $24-1,50-52$ & t & e & 100 & & & & & $\epsilon$ & & & & & $\epsilon$ & & & \\
\hline $24-2,13-15$ & e & $t$ & 100 & & & & & e & & & & & - & & & \\
\hline $24-3,31-34$ & & $t$ & 100 & & & & & t & & & & & $\epsilon$ & & & \\
\hline $25-1,32-35$ & & $\epsilon$ & 100 & & & & & ? & & & & & $\epsilon$ & & & \\
\hline $26-1$ & 5 & e & 100 & & & & & e & & & $?$ & & $\epsilon$ & & & \\
\hline
\end{tabular}


APPENDIX II

Table 1. (Continued).

\begin{tabular}{|c|c|c|c|c|c|c|c|}
\hline $\begin{array}{l}\text { Core-Section } \\
\text { (interval or } \\
\text { level in } \mathrm{cm} \text { ) }\end{array}$ & Clays & Quartz & Calcite & Dolomite & $\begin{array}{l}\text { Plagio- } \\
\text { clase }\end{array}$ & $\begin{array}{l}\text { K-feld- } \\
\text { spars }\end{array}$ & Opal-CT \\
\hline & & & & & & & \\
\hline $27-1,17-19$ & 5 & $\epsilon$ & 95 & & & & \\
\hline $27-1,17-19$ & & $\epsilon$ & 80 & & & & 20 \\
\hline $28-1,71-73$ & 10 & e & 90 & & & & \\
\hline $28-2,23-25$ & 5 & $\epsilon$ & 90 & & & & 5 \\
\hline $28-3,4-6$ & 5 & e & 90 & & & & 5 \\
\hline $29-1,17-19$ & 5 & ¿ & 90 & & & & 5 \\
\hline $34-1,11-14$ & 30 & 15 & 35 & & & & 15 \\
\hline $34-1,19-21$ & 30 & 20 & 40 & & & & 5 \\
\hline $37-2,27-29$ & 35 & 15 & 40 & & & & 5 \\
\hline $\begin{array}{l}38, \mathrm{CC} \\
39-1,24-25\end{array}$ & $\begin{array}{l}25 \\
35\end{array}$ & is & $\begin{array}{l}55 \\
35\end{array}$ & & & & 5 \\
\hline $\begin{array}{l}39-1,24-25 \\
40-1,6-8\end{array}$ & 30 & 15 & 35 & & & & $\begin{array}{l}5 \\
5\end{array}$ \\
\hline $42-1,60-61$ & 30 & 15 & 45 & & & & $\epsilon$ \\
\hline $43-1,83-86$ & 15 & et & 85 & & & & $t$ \\
\hline $43-2,68-69$ & 15 & $e^{+}$ & 85 & & & & \\
\hline $43-3,55-58$ & 30 & 10 & 30 & & & & 5 \\
\hline $43-4,40-41$ & 35 & 20 & 35 & & & & 5 \\
\hline $44-2,110-116$ & 15 & $\therefore$ & 85 & & & & $t$ \\
\hline $45-1,56-58$ & 30 & 20 & 45 & & & & e \\
\hline $\begin{array}{l}45-3,109-113 \\
45, C C\end{array}$ & $\begin{array}{l}30 \\
25\end{array}$ & $\begin{array}{l}20 \\
10\end{array}$ & $\begin{array}{l}45 \\
65\end{array}$ & & & & \\
\hline $\begin{array}{l}45, C C \\
46-1,6-8\end{array}$ & 35 & 15 & 40 & & & & e \\
\hline $46-1,23-24$ & 30 & 20 & 40 & & & & $\epsilon$ \\
\hline $47-2,143-149$ & 35 & 25 & 30 & & & & 10 \\
\hline $52-1,96-98$ & $\epsilon$ & 20 & . & 80 & & & \\
\hline $53-1,141-144$ & & 05 & 30 & & & & \\
\hline $\begin{array}{l}53-2,13-15 \\
53-2,136-139\end{array}$ & & $\begin{array}{l}05 \\
05\end{array}$ & $\begin{array}{l}35 \\
30\end{array}$ & & & & \\
\hline $54-1,8-10$ & & 05 & 25 & & & & \\
\hline $55-1,40-43$ & & 10 & 60 & & & & \\
\hline $55-3,74-77$ & & 10 & 70 & & & & \\
\hline $55-5,57-60$ & & 10 & 55 & & & & \\
\hline $\begin{array}{l}56-2,68-72 \\
56-4,32-39\end{array}$ & & $\begin{array}{l}25 \\
30\end{array}$ & $\begin{array}{l}55 \\
35\end{array}$ & & & & \\
\hline $57-2,144-146$ & & 30 & 25 & & & & \\
\hline $57-5,26-29$ & & 30 & 20 & & & & \\
\hline $58-3,79-81$ & & 20 & 25 & & & & \\
\hline $58-4,51-54$ & & 25 & 20 & & & & \\
\hline $58-5,85-87$ & & 30 & 25 & & & & \\
\hline $58-6,86-89$ & & 20 & 20 & & & & \\
\hline $59-2,42-44$ & & 25 & 20 & & & & \\
\hline $59-3,106-108$ & & 25 & 35 & & & & \\
\hline $60-1,48-50$ & & 30 & 20 & & & & \\
\hline $\begin{array}{l}60-3,102-105 \\
61-2,19-22\end{array}$ & & $\begin{array}{l}25 \\
25\end{array}$ & $\begin{array}{l}20 \\
25\end{array}$ & & & & \\
\hline $61-4,11-13$ & & 25 & 20 & & & & \\
\hline $72-1,66-68$ & & & 20 & & & & \\
\hline $72-2,100-102$ & & & 25 & & & & \\
\hline $73-1,31-33$ & & 10 & 10 & & & & \\
\hline $78-1,28-30$ & & $\begin{array}{l}50 \\
25\end{array}$ & $\begin{array}{l}05 \\
10\end{array}$ & & & & \\
\hline $\begin{array}{l}79-1,88-91 \\
80-1,30-34\end{array}$ & & $\begin{array}{l}25 \\
40\end{array}$ & $\begin{array}{l}10 \\
15\end{array}$ & & & & \\
\hline $81-1,27-29$ & & 30 & 05 & & & & \\
\hline $82-1,8-10$ & & 50 & 15 & & & & \\
\hline $82-1,139-141$ & & 50 & 10 & & & & \\
\hline $82-2,13-15$ & & 40 & 10 & & & & \\
\hline $82-2,68-70$ & & 30 & 10 & & & & \\
\hline $84-1,48-50$ & & 25 & 05 & & & & \\
\hline $86-1,51-53$ & & 30 & 10 & & & & \\
\hline $86-1,139-141$ & & 30 & 15 & & & & \\
\hline $86-2,58-59$ & & 25 & 05 & & & & \\
\hline $86-2,111-113$ & & 25 & 15 & & & & \\
\hline $86-3,42-43$ & & 25 & 65 & & & & \\
\hline $88-1,1-3$ & & 10 & 10 & & & & \\
\hline $88-1,112-115$ & & 10 & 25 & & & & \\
\hline $88-1,121-123$ & & 40 & 05 & & & & \\
\hline $88-2,3-6$ & & 15 & i & & & & \\
\hline $\begin{array}{l}88-2,43-45 \\
90-1,25-28\end{array}$ & & 15 & $\begin{array}{l}05 \\
05\end{array}$ & & & & \\
\hline $\begin{array}{l}90-1,25-28 \\
90-2,108-110\end{array}$ & & $\begin{array}{l}50 \\
40\end{array}$ & $\begin{array}{l}05 \\
05\end{array}$ & & & & \\
\hline $90-3,1-3$ & & 30 & 05 & & & & \\
\hline $90, \mathrm{CC}(17-19)$ & & 25 & 05 & & & & \\
\hline $91-2,21-23$ & & 50 & 10 & & & & \\
\hline $91, \mathrm{CC}$ & & 40 & 05 & & & & \\
\hline & & & & & & & \\
\hline $1-1,53-55$ & 20 & $\epsilon$ & 80 & & & & \\
\hline $1-1,126-128$ & $\epsilon$ & $\epsilon$ & 100 & & & & \\
\hline $1-3,55$ & 5 & $\epsilon$ & 95 & $?$ & & & \\
\hline $1-3,67-68$ & 15 & $\epsilon$ & 85 & & & & \\
\hline $1-4,77-80$ & 5 & $\epsilon$ & 95 & ? & & & \\
\hline $1-6,104-106$ & 30 & $\epsilon$ & 70 & & & & \\
\hline $2-3,28-29$ & 30 & $\epsilon$ & 70 & & $?$ & & \\
\hline $2-3,101-103$ & & & & & & & \\
\hline $3-1,132-133$ & 10 & $\epsilon$ & 90 & & & & \\
\hline $3-2,19-21$ & is & ' & 100 & & & ? & \\
\hline $4-1,75-78$ & 15 & e & 80 & & & & \\
\hline $5-1,45-47$ & & & & & & & \\
\hline 5-2, 9-11 & 15 & $t$ & 85 & & & & \\
\hline $5-2,30-32$ & 5 & $\epsilon$ & 95 & & & & \\
\hline $5-2,73-76$ & 5 & $t$ & 95 & & & & \\
\hline $5-3,81-83$ & 5 & E & 95 & & & & \\
\hline $5-4,37-38$ & $\epsilon$ & $\epsilon$ & 100 & & & & \\
\hline $7-1,30-32$ & e & $\epsilon$ & 100 & & & & \\
\hline $7-1,148-150$ & E & $e$ & 95 & & & & \\
\hline $7-2,56-58$ & 15 & E & 85 & & & & \\
\hline & & & & & & & \\
\hline
\end{tabular}


Table 1. (Continued).

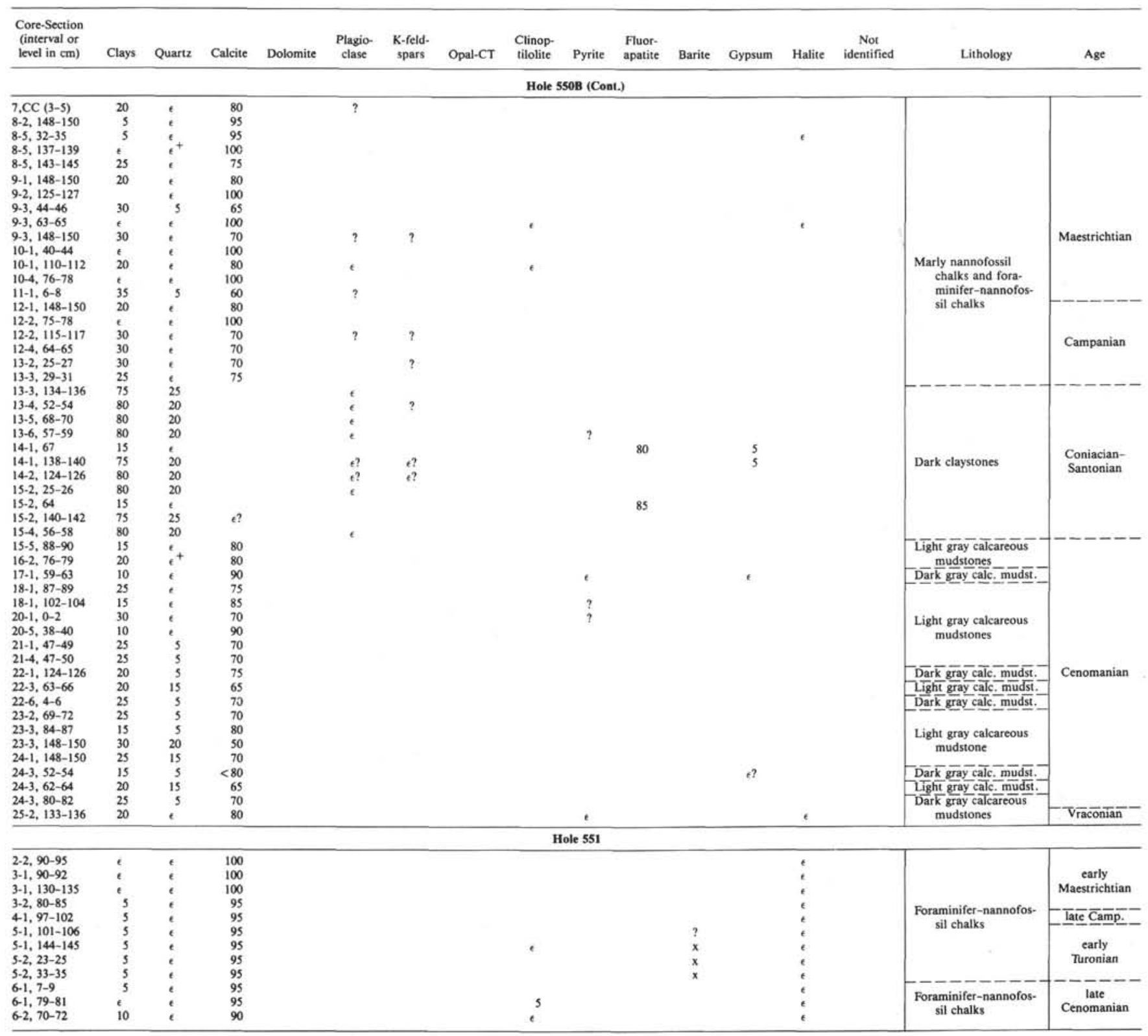

Note: $e=$ trace, $x=$ present. 
Table 2. X-ray analysis of $<2 \mu \mathrm{m}$ fraction.

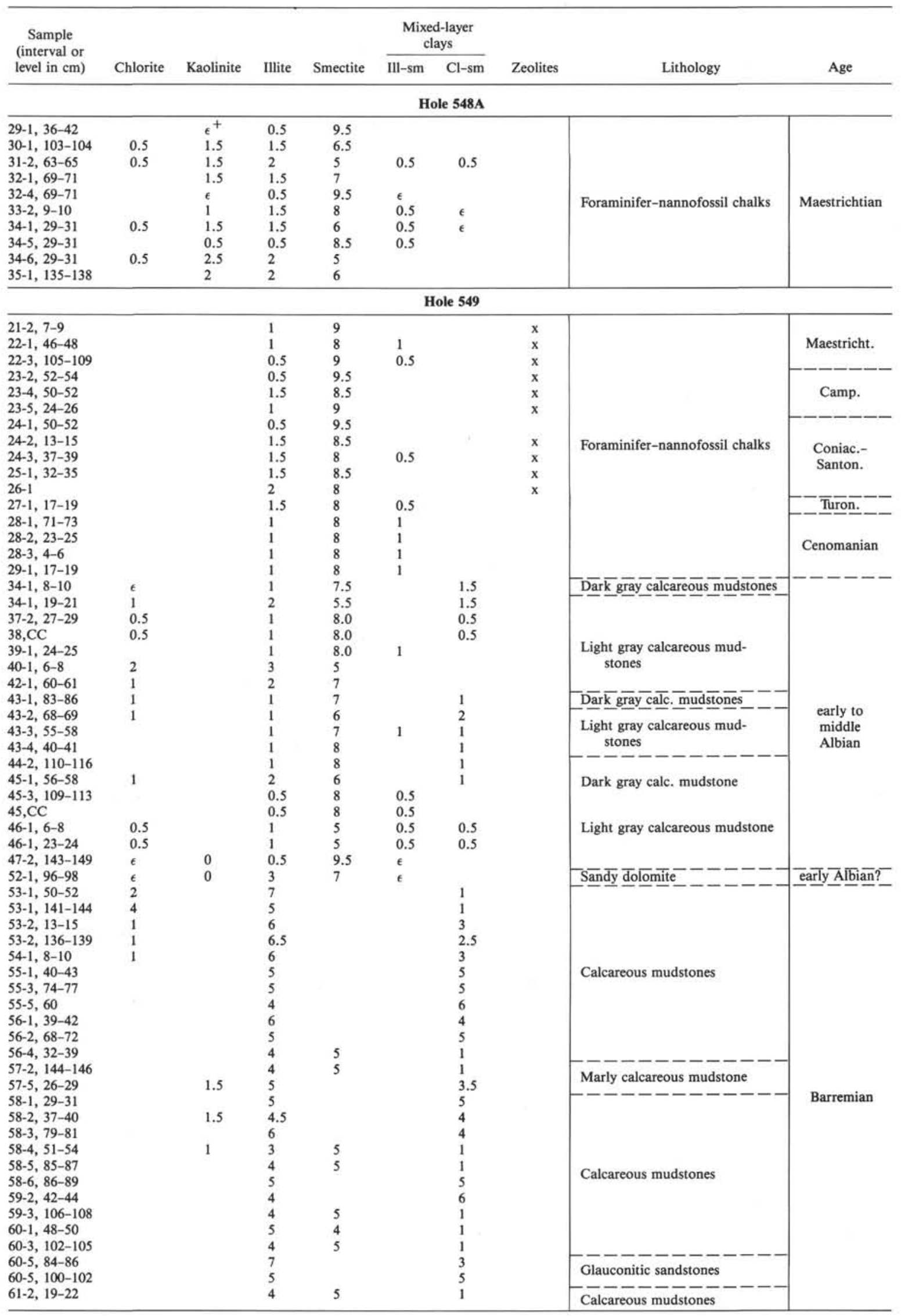


Table 2. (Continued).

\begin{tabular}{|c|c|c|c|c|c|c|c|c|c|}
\hline \multirow{2}{*}{$\begin{array}{c}\text { Sample } \\
\text { (interval or } \\
\text { level in } \mathrm{cm} \text { ) }\end{array}$} & \multirow[b]{2}{*}{ Chlorite } & \multirow[b]{2}{*}{ Kaolinite } & \multirow[b]{2}{*}{ Illite } & \multirow[b]{2}{*}{ Smectite } & \multicolumn{2}{|c|}{$\begin{array}{c}\text { Mixed-layer } \\
\text { clays }\end{array}$} & \multirow[b]{2}{*}{ Zeolites } & \multirow[b]{2}{*}{ Lithology } & \multirow[b]{2}{*}{ Age } \\
\hline & & & & & Ill-sm & $\mathrm{Cl}$-sm & & & \\
\hline \multicolumn{10}{|c|}{ Hole 549 (Cont.) } \\
\hline $61-2,129-131$ & 0.5 & & 3.5 & 6 & & & & & \\
\hline $61-4,11-13$ & & & 4 & 5 & & 1 & & Calcareous mudstones. & \\
\hline $\begin{array}{l}72-1,66-68 \\
72-2,100-102\end{array}$ & & & $\begin{array}{l}4 \\
5\end{array}$ & 6 & & 5 & & Bioclastic limestones & \\
\hline $73-1,31-33$ & & & 4.5 & 1.5 & & 1 & & Calcareous mudstone - & \\
\hline $74-3,44-46$ & & 0.5 & 5.5 & & & 4 & & Siltstone- & \\
\hline $75, \mathrm{CC}, 24-26$ & 1.5 & 2.5 & 5 & & 1 & & & Limestone & \\
\hline $78-1,28-30$ & 3.5 & 1 & 5.5 & & & & & Sandstone & \\
\hline $79-1,88-91$ & 2.5 & 2.5 & 4 & & 1 & & & & \\
\hline $80-1,30-34$ & 1 & 3 & 4 & & 2 & & & Calcareous mudstones & \\
\hline $\begin{array}{l}81-1,27-29 \\
82-1,8-10\end{array}$ & 1 & $\begin{array}{l}2.5 \\
3\end{array}$ & $\begin{array}{l}5.5 \\
5\end{array}$ & & $\begin{array}{l}1 \\
1\end{array}$ & & & Silty limestone $\overline{-}-------$ & \\
\hline $82-1,47-49$ & & 4 & 6 & & & & & - -1 & \\
\hline $82-1,139-141$ & & 3.5 & 4.5 & & 2 & & & & \\
\hline $82-2,13-15$ & & 2 & 6 & & 2 & & & Calcareous mudstones & \\
\hline $82-2,68-70$ & & 4 & 4 & & 2 & & & 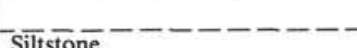 & \\
\hline $\begin{array}{l}84-1,48-50 \\
85-3,28-30\end{array}$ & 4 & $\begin{array}{l}2 \\
1\end{array}$ & $\begin{array}{l}6 \\
4\end{array}$ & & 2 & 1 & & $\begin{array}{l}\text { Siltstone } \\
\text { Sandstone } \\
\text { Sond }\end{array}$ & \\
\hline $86-1,51-53$ & i & 3 & 4.5 & & 1.5 & & & ㄴ- - - - - - & \\
\hline $86-1,139-141$ & 2 & 3 & 4 & & 1 & & & & Barremian \\
\hline $86-2,58-59$ & 3 & 3 & 4 & & & & & Calcareous mudstones & \\
\hline $86-2,111-113$ & 1 & 3 & 4 & & 2 & & & Site- - - - - - - - - - & \\
\hline $86-3,42-43$ & 1 & 3 & 4 & & 2 & & & Silty limestone $-----\cdots$ & \\
\hline $88-1,1-3$ & 1 & 2 & 4 & & 3 & & & & \\
\hline $88-1,112-115$ & 2.5 & 1.5 & 5 & & 1 & & & Sandstones & \\
\hline $88-1,121-123$ & 1 & 2.5 & 4.5 & & 2 & & & & \\
\hline $88-2,3-6$ & & 2.5 & 5 & & & $\begin{array}{l}2.5 \\
1\end{array}$ & & Calcareous mudstone & \\
\hline $88-2,43-45$ & & 2.5 & 6.5 & & & & & Silty limestone & \\
\hline $\begin{array}{l}88, \mathrm{CC} \\
89-2,9-11\end{array}$ & $\begin{array}{l}3 \\
2\end{array}$ & $\begin{array}{l}2.5 \\
2.5\end{array}$ & $\begin{array}{l}2.5 \\
5.5\end{array}$ & & & & & & \\
\hline $90-1,25-28$ & 1 & 3 & 5 & & 1 & & & Calcareous mudstones & \\
\hline $90-2,107-110$ & & 2.5 & 4.5 & & & 3 & & Sandstones & \\
\hline $90-3,0-3$ & 1 & 3 & 4 & & 2 & & & & \\
\hline $90, \mathrm{CC}, 17-19$ & & 2 & 5 & & & 3 & & Siltstone $-1-----$ & \\
\hline $91-1,44-45$ & 2 & 2 & 5 & & 1 & & & 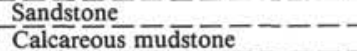 & \\
\hline $91-2,21-23$ & 1 & 2 & 6 & & 1 & & & Calcareous mudstone & \\
\hline $91, \mathrm{CC}$ & 1 & 2 & 6.5 & & & 2.5 & & Sandstone - Calcareous mudstone- & \\
\hline $92-1,8-10$ & 2 & 2.5 & 4.5 & & 1 & & & Calcareous mudstone $-\ldots$ & \\
\hline $\begin{array}{l}92-1,106-108 \\
93-1,72-75\end{array}$ & $\begin{array}{l}1 \\
1\end{array}$ & $\begin{array}{l}1 \\
1\end{array}$ & $\begin{array}{l}8 \\
7\end{array}$ & & & 1 & & Sandstones & \\
\hline & & & & & & le 550B & & & \\
\hline $1-1,53-55$ & 1 & 3 & 2 & 3 & & 1 & & & \\
\hline $1-1,126-128$ & & $\epsilon^{+}$ & 0.5 & 9.5 & & $\epsilon$ & & & \\
\hline $1-3,55$ & & 3 & 1.5 & 4 & & 1.5 & & & \\
\hline $1-3,67-68$ & & 3 & 2 & 4 & & 1 & & & \\
\hline $1-4,77-80$ & & $\epsilon$ & 0.5 & 9.5 & & & $\mathrm{x}$ & & \\
\hline $\begin{array}{l}1-6,104-106 \\
2-1,57-59\end{array}$ & & 1 & 0.5 & 8.5 & & $\epsilon$ & & & \\
\hline $2-3,28-29$ & & 2 & 1 & 7 & & $\epsilon$ & & & \\
\hline $2-3,101-103$ & & 2 & 1 & 7 & & $\epsilon$ & & & \\
\hline $3-1,132-133$ & 2 & 2 & 2 & 1 & & 3 & & & \\
\hline $3-2,19-21$ & & 2.5 & 1.5 & 5 & & 1 & & & \\
\hline $4-1,75-78$ & 2 & 2 & 2 & 2 & & 2 & & & \\
\hline $5-1,45-47$ & 2 & & 2 & 4 & & 2 & & & \\
\hline $5-2,9-11$ & & 2 & 2 & 5 & & 1 & & & \\
\hline $5-2,30-32$ & & 1 & 1 & 8 & & $\epsilon$ & & Marly nannofossil chalks and & \\
\hline $\begin{array}{l}5-2,40-43 \\
5-3,81-83\end{array}$ & $\epsilon$ & $\begin{array}{l}1.5 \\
\epsilon\end{array}$ & $\begin{array}{l}1.5 \\
\epsilon\end{array}$ & $\begin{array}{r}7 \\
10\end{array}$ & & & & foraminifer-nannofossil & Maestrichtian \\
\hline $5-4,37-38$ & & 1 & 1 & 7.5 & & 0.5 & & chalks & \\
\hline $7-1,30-32$ & & 1 & 0.5 & 8.5 & & $\epsilon$ & & & \\
\hline $7-1,148-150$ & & 1.5 & 1 & 7.5 & & $\epsilon$ & & & \\
\hline $7-2,56-58$ & & 2 & 2 & 4 & 2 & $\epsilon$ & & & \\
\hline $7-3,13-15$ & $\epsilon$ & 2 & 1 & 6.5 & & 0.5 & & & \\
\hline $7, \mathrm{CC}(4-5)$ & 1 & 2 & 2 & 4 & 1 & & & & \\
\hline $\begin{array}{l}8-2,148-150 \\
8-3,49-50\end{array}$ & 1 & 1 & 1 & 5 & & 2 & & & \\
\hline $8-5,32-35$ & 1 & 1 & 1 & 5 & & 2 & & & \\
\hline $8-5,137-139$ & 0.5 & 0.5 & 1 & 7 & & 1 & & & \\
\hline $8-5,143-145$ & 0.5 & 0.5 & 1 & 7 & & 1 & & & \\
\hline $9-1,148-150$ & & 2 & 2 & 4 & & 2 & & & \\
\hline $9-2,125-127$ & & 1 & 1 & 5 & 3 & & & & \\
\hline $9-3,44-46$ & 1 & 3 & 1 & 2 & 1 & 2 & & & \\
\hline $9-3,63-65$ & & 1.5 & 1.5 & 6 & & 1 & & & \\
\hline
\end{tabular}


Table 2. (Continued).

\begin{tabular}{|c|c|c|c|c|c|c|c|c|c|}
\hline \multirow{2}{*}{$\begin{array}{l}\text { Sample } \\
\text { (interval or } \\
\text { level in } \mathrm{cm} \text { ) }\end{array}$} & \multirow[b]{2}{*}{ Chlorite } & \multirow[b]{2}{*}{ Kaolinite } & \multirow[b]{2}{*}{ Illite } & \multirow[b]{2}{*}{ Smectite } & \multicolumn{2}{|c|}{$\begin{array}{l}\text { Mixed-layer } \\
\text { clays }\end{array}$} & \multirow[b]{2}{*}{ Zeolites } & \multirow[b]{2}{*}{ Lithology } & \multirow[b]{2}{*}{ Age } \\
\hline & & & & & III-sm & $\mathrm{Cl}$-sm & & & \\
\hline \multicolumn{10}{|c|}{ Hole 550B (Cont.) } \\
\hline $9-3,148-150$ & & 3 & 2 & 3 & & 2 & & \multirow{11}{*}{$\begin{array}{l}\text { Marly nannofossil chalks and } \\
\text { foraminifer-nannofossil } \\
\text { chalks }\end{array}$} & \multirow{5}{*}{ Maestrichtian } \\
\hline $10-1,40-44$ & 1 & 1 & 1 & 7 & & & & & \\
\hline $10-1,110-112$ & 1 & 2 & 2 & 3 & & 2 & & & \\
\hline $10-4,76-78$ & & 1 & 1 & 7 & & 1 & & & \\
\hline $11-1,6-8$ & & 1 & 1 & 7 & 0.5 & 0.5 & & & \\
\hline $11-2,63-65$ & & 0.5 & 0.5 & 8 & 1 & & & & \multirow{6}{*}{ Campanian } \\
\hline $12-1,148-150$ & & & & 10 & & & & & \\
\hline $\begin{array}{l}12-2,75-77 \\
12-2,115-117\end{array}$ & & & $\epsilon$ & 10 & & & & & \\
\hline $\begin{array}{l}12-2,115-117 \\
12-4,64-65\end{array}$ & & 0.5 & $\begin{array}{l}\epsilon \\
1\end{array}$ & $\begin{array}{l}10 \\
8.5\end{array}$ & & & & & \\
\hline $13-2,25-27$ & & 1 & 1 & 7 & 1 & $\epsilon$ & & & \\
\hline $13-3,29-31$ & & 0.5 & 0.5 & 9 & & & & & \\
\hline $13-3,134-136$ & & 1 & 1 & 8 & & & & \multirow{8}{*}{ Dark claystones } & \multirow{8}{*}{$\begin{array}{l}\text { Coniac.- } \\
\text { Santonian }\end{array}$} \\
\hline $13-4,52-54$ & & $\epsilon$ & 1 & 9 & & & & & \\
\hline $13-5,68-70$ & & & 0.5 & 9.5 & & & & & \\
\hline $13-6,57-59$ & & $\epsilon$ & $\epsilon$ & 10 & & $\epsilon$ & & & \\
\hline $15-2,25-26$ & $\epsilon$ & & 0.5 & 9.5 & $\epsilon$ & ( & & & \\
\hline $15-2,64$ & & & & 10 & & & & & \\
\hline $15-2,140-142$ & $\epsilon$ & & 0.5 & 9.5 & $\epsilon$ & $\epsilon$ & & & \\
\hline $15-4,56-58$ & \multirow[t]{8}{*}{$\epsilon$} & & 0.5 & 9.5 & $\epsilon$ & & & & \\
\hline $15-5,88-90$ & & & 0.5 & 9.5 & $\epsilon$ & & & Light-gray calc. mudst. & ----- \\
\hline $16-2,76-79$ & & & 0.5 & 9 & 0.5 & & & & \\
\hline $17-1,59-63$ & & & 0.5 & 9.5 & & & & Dark gray calc. mudst. & \\
\hline $18-1,87-89$ & & & 0.5 & 9.5 & $\epsilon$ & & & & \\
\hline $18-1,102-104$ & & & 0.5 & 9.5 & $\epsilon$ & & & & \\
\hline $20-1,0-2$ & & & $\epsilon^{+}$ & 10 & & & & & \\
\hline $20-5,38-40$ & & $\epsilon$ & 0.5 & 9.5 & $\epsilon$ & & & Light gray calc. mudst. & \\
\hline $21-1,47-49$ & $\epsilon ?$ & $\epsilon ?$ & 0.5 & 9.5 & $\epsilon$ & & & & \\
\hline $21-4,47-50$ & & $\epsilon$ & 0.5 & 9.5 & $\epsilon$ & & & & \\
\hline $22-1,124-126$ & $\epsilon$ & $\epsilon$ & 0.5 & 9 & 0.5 & & & Dark gray calc. mudst. & \\
\hline $22-3,63-66$ & & & & & & & & Light gray calc. mudst. & Cenomanian \\
\hline $22-6,4-6$ & $\epsilon$ & $\epsilon ?$ & 1 & 7.5 & 1 & 0.5 & & Dark gray calc. mudst. & \\
\hline $22-6,63-66$ & & & No & nalysis ava & & & & & \\
\hline $23-2,69-72$ & $\epsilon$ & $\epsilon ?$ & 1.5 & 6 & 1.5 & 1 & & & \\
\hline $23-3,84-87$ & & $\epsilon$ & 1 & 1 & 1 & 1 & & & \\
\hline $23-3,148-150$ & $\epsilon$ & $\epsilon ?$ & 1 & 8 & 1 & & & Light gray calc. mudst. & \\
\hline $24-1,148-150$ & & $\epsilon$ & 1 & 7.5 & 1.5 & $\epsilon$ & & & \\
\hline $24-3,52-54$ & 1 & $\epsilon$ ? & 2 & 7 & & & & & \\
\hline $24-3,62-64$ & $\epsilon$ & $?$ & 1 & 7.5 & 1.5 & $\epsilon^{+}$ & & 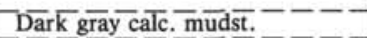 & \\
\hline $24-3,80-82$ & 1 & ? & 1.5 & 6 & 1.5 & & & Light gray calc. mudst. & \\
\hline $25-2,133-136$ & 0.5 & ? & 1.5 & 6.5 & 1.5 & & & Dark gray calc. mudst. - & - Vraconian \\
\hline & & & & & & ole 551 & & & \\
\hline $2-2,90-95$ & & & $\epsilon$ & 10 & & & & & \\
\hline $3-1,90-92$ & & & 0.5 & 9 & & 0.5 & & & early \\
\hline $3-1,130-135$ & & & 0.5 & 9.5 & $\epsilon$ & & & & Maestrichtian \\
\hline $3-2,80-85$ & & & $\epsilon^{+}$ & 9.5 & 0.5 & & & & \\
\hline $4-1,97-102$ & & & 0.5 & 8 & 1.5 & & & & late Camp. \\
\hline $5-1,101-106$ & & & 1.5 & 8.5 & & & $\mathbf{x}$ & Foraminifor - & \\
\hline $5-1,144-145$ & & & 0.5 & 9 & 0.5 & & & Foraminifer-nannofossil chalks & early \\
\hline $5-2,23-25$ & & & 1.5 & 8.5 & & & $\mathrm{x}$ & & Turonian \\
\hline $5-2,33-35$ & & & 1.5 & 8.5 & & & $\mathrm{x}$ & & \\
\hline $6-1,7-9$ & & & 3 & 4 & 3 & & & & \\
\hline $6-1,79-81$ & & & 2 & 2 & 6 & & & & $\begin{array}{l}\text { late } \\
\text { Cenomanian }\end{array}$ \\
\hline $6-2,70-72$ & & & 1 & 8 & 1 & & $\mathrm{x}$ & & \\
\hline
\end{tabular}

Note: Quantities are totaled to $10 . \epsilon=$ trace, $x=$ present. IIl-sm $=$ illite-smectite mixed-layer clay; $\mathrm{Cl}$-sm $=$ chlorite-smectite mixed-layer clay 


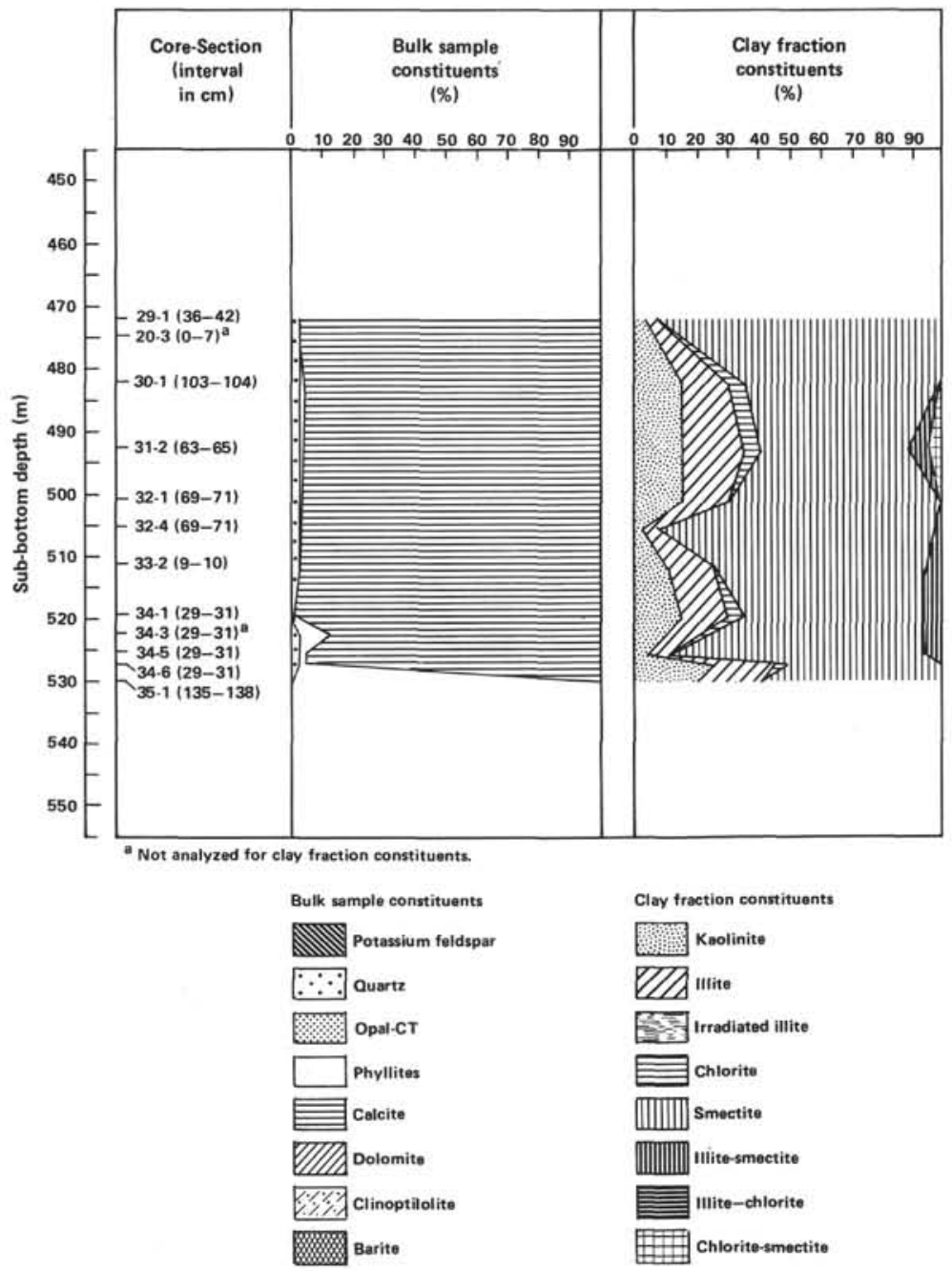

Figure 1. Bulk sample and clay fraction constituents of sediments from Hole 548A (Cores 29-34). 


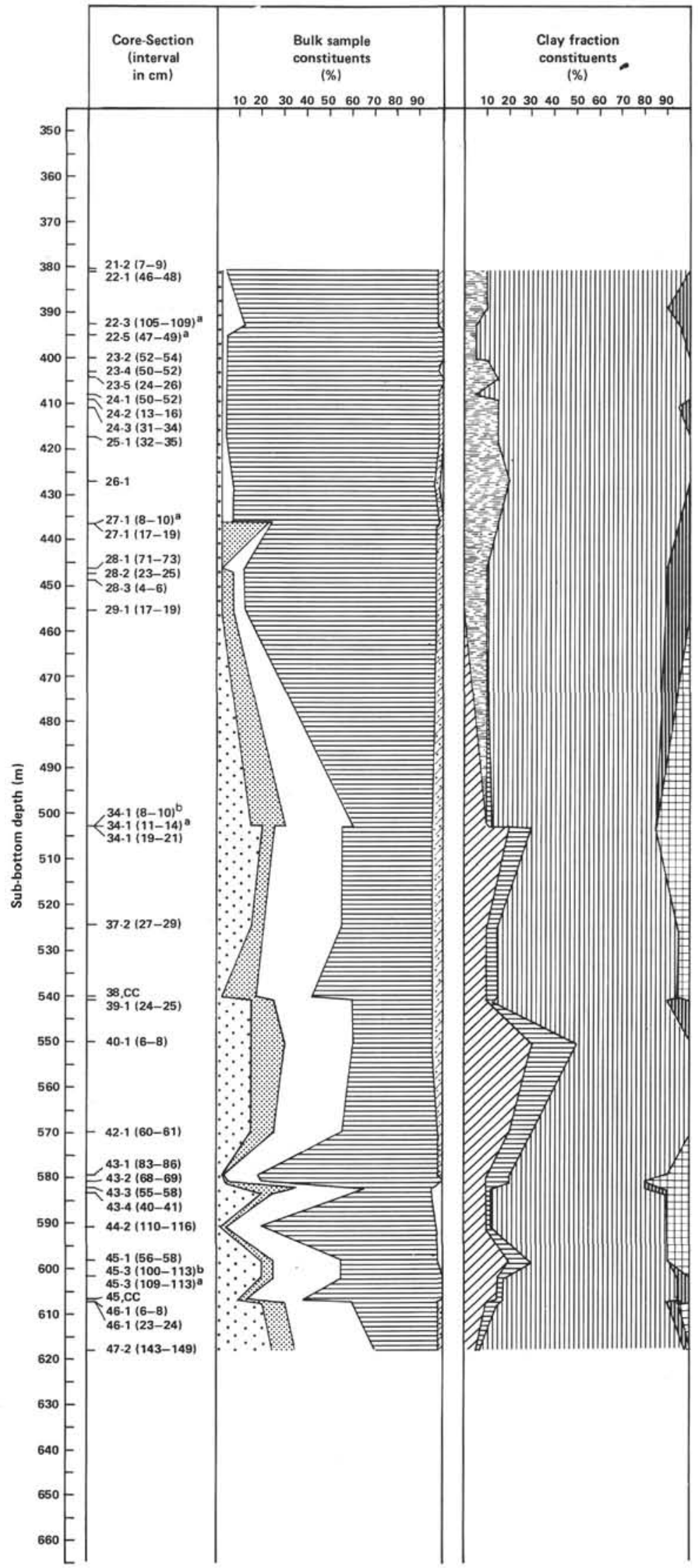

Figure 2. Bulk sample and clay fraction constituents of sediments from Hole 549 (Cores 21-91). Legend as in Fig. 1. 


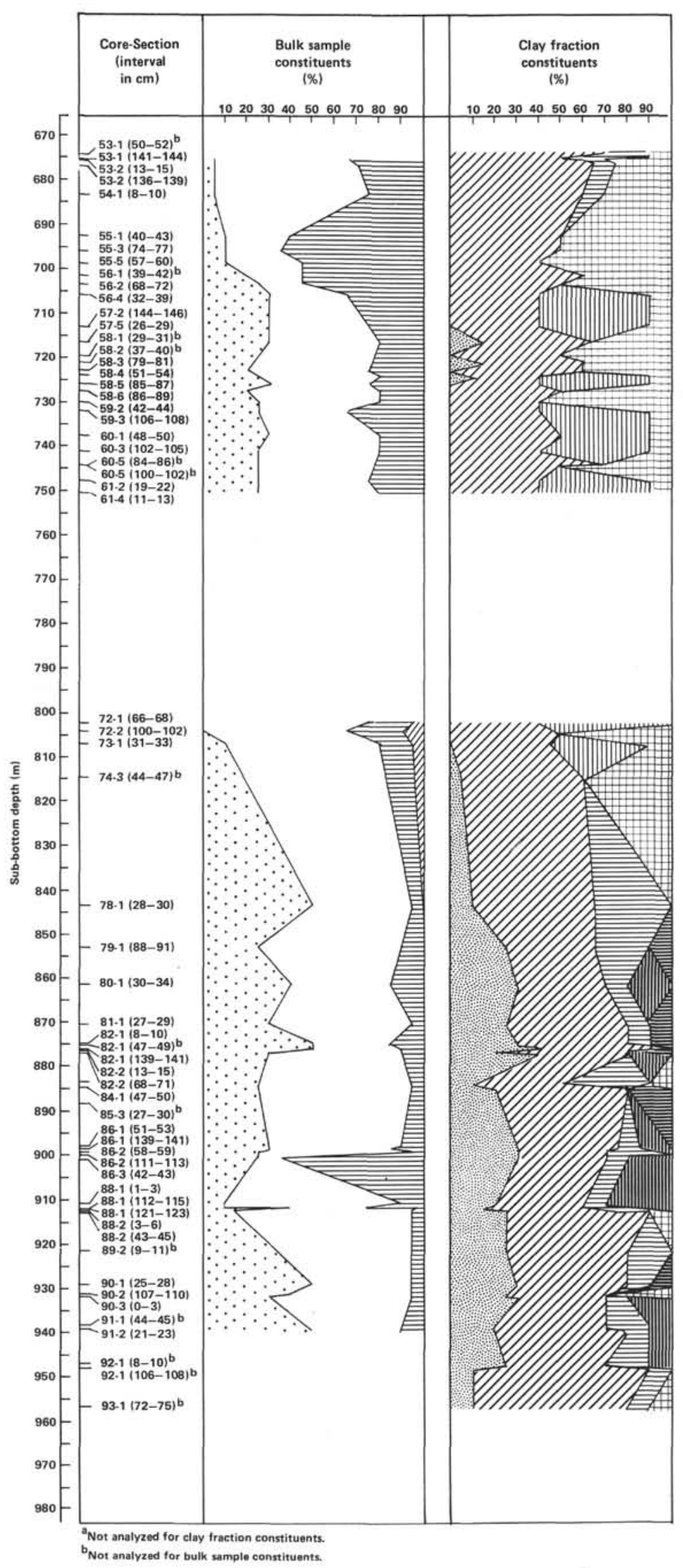

Figure 2. (Continued). 


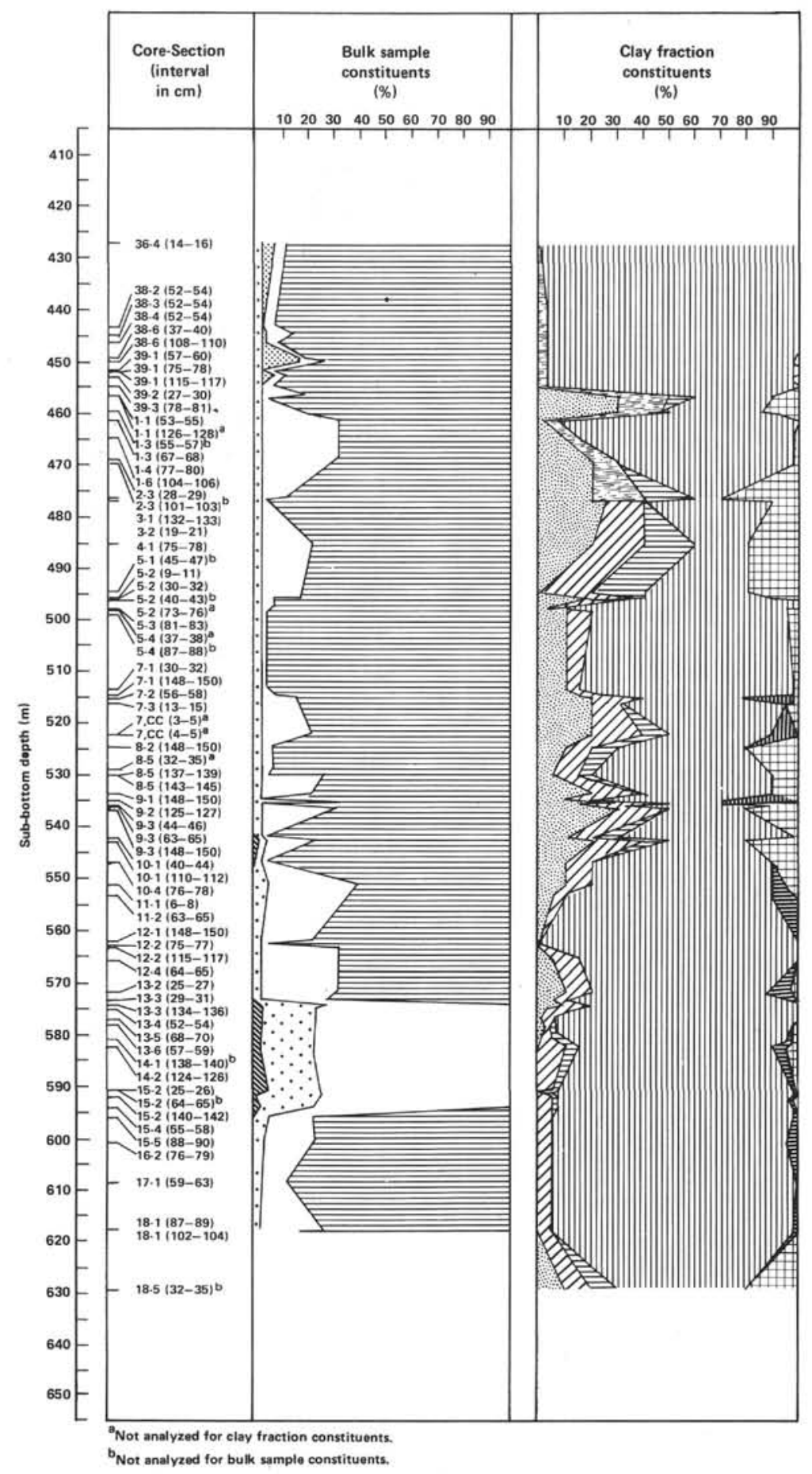

Figure 3. Bulk sample and clay fraction constituents of sediments from Holes 550 (Cores 36-39) and 550B (Cores 1-18). Legend as in Fig. 1. 